\begin{tabular}{|c|c|c|}
\hline Beitr. Ent. & Keltern & ISSN 0005-805X \\
\hline $\mathbf{5 7}(2007) 1$ & S. $151-159$ & 30.06 .2007 \\
\hline
\end{tabular}

\title{
Ein kurzer Beitrag zum Massenwechsel der Gemeinen Kiefern- buschhornblattwespe, Diprion pini (L., 1758) in Beytepe bei Ankara
}

\section{(Hymenoptera: Diprionidae)}

Mit 7 Tabellen

Ilhami KiziroĞLu und Levent Turan

\section{Zusammenfassung}

In einem Untersuchungsgebiet bei Ankara / Türkei wurden fast alle Kiefern (Pinus nigra) von der Kiefernbuschhornblattwespe, Diprion pini, befallen. 14,0 \% der Bäume wurden kahl gefressen.

D. pini hatte während der Sommergeneration in Beytepe 13,7 \% der Nadeln mit Eiern belegt. Die Anzahl der Eier pro Gelege schwankte zwischen 16-25, ihr prozentualer Wert betrug 30,4\%. Dabei hatte die Nadellänge keinen Einfluss auf die Eiablage.

Es wurde keine Parasitierung an Eiern bzw. an Larven festgestellt. Die Parasitierungswerte bei männlichen Kokons wurden mit 1,1\%, bei weiblichen dagegen mit 14,1\% ermittelt.

Ohne Berücksichtigung der nicht ausgeschlüpften bzw. parasitierten Kokons betrug der Weibchenanteil in der Beytepe-Population des Schädlings 54,1 \%. Der Unterschied in der Breite der weiblichen und männlichen Kokons von $D$. pini ist mit einer Sicherheit von $\mathrm{p}>0,05$ signifikant, bei Kokonlänge dagegen nicht.

\section{Schlüsselwörter}

Diprion pini, Populationsökologie, Massenwechsel, Kokongröße, Parasitierung

\section{Summary}

The Common Pine Sawfly, D. pini L., has damaged all pine (Pinus nigra L.) trees in various sections and completely destroyed $14,0 \%$ of them. The number of eggs encountered in an egg stack is between 16-25, and the frequency rate of this is $30,4 \%$. Here the role of the needle is important. The summer generation of $D$. pini population in Beytepe left eggs on $13,7 \%$ of the needles.

The parasitization rate of Cocoons is $1,1 \%$ for male Cocoons and $14,1 \%$ for female Cocoons. Unless unopened and parasitized Cocoons are ignored, the female rate reaches $54,1 \%$. When the widths of female and male Cocoons are compared, female Cocoons are significantly wider with a value of $p>0,05$. The difference between the lengths of female and male Cocoons is not significant.

\section{Keywords}

Population ecology, population dynamic, widths of cocoons, Coccons parasitized 


\section{Einleitung}

Aus der Literatur ging hervor, dass die Gemeine Kiefernbuschhornblattwespe, Diprion pini (L., 1758), schon an mehreren Stellen der Türkei in größerem Umfang aufgetreten ist (ACATAY 1969, ÇANAKÇıoĞLu 1998 und ŞıMŞEK 2004). D. pini hat in der Türkei, wie in den meisten europäischen Ländern zwei Generationen (SChWenke 1964, Pschorn-Walcher 1982, EichHorn 1983a, 1983b, Auger-Rozenberg u. a. 1998). Sie hat variable Generationsverhältnisse und kann bis zu 5 Jahre Überliegen, das heißt im Kokon Überdauern ohne auszuschlüpfen (BogENsCHÜTZ u. a. 1982, PSCHORN-WALCHER 1982, EICHHORN 1983a).

Sie gilt in verschiedenen Bereichen von Europa als wichtigster Forstschädling in Kiefernkulturen (Thalenhorst 1952, 1954, Kangas 1963, Schwenke 1960, 1964, Urban 1965, PschornWalcher 1982, EichHorn 1980, 1981, 1983a, 1983b, Sharov u. a. 1993, Auger-Rozenberg u. a. 1998).

In den Jahren 2000 und 2002 trat in einer 30 jährigen Kiefern- und Zedernkultur bei Ankara Diprion pini in so großer Befallsstärke auf, dass der größte Teil der Bäume kahl gefressen wurde. Daraus resultiert die Aufgabe, einige biologischen Daten dieses Forstschädlings für weitere populationsbiologische Untersuchungen zu gewinnen.

\section{Material und Methoden}

Das Untersuchungsgebiet befindet sich in der Ortschaft Beytepe am nordwestlichen Stadtrand von Ankara. Im Untersuchungsgebiet, das ca. $10 \mathrm{~km}^{2}$ groß ist und hauptsächlich mit Schwarzkiefern (Pinus nigra) und Libanesischen Zedern (Cedrus libani) bewachsen ist, wurden insgesamt 298 Kiefern in einer Teilfläche von ca. $1-2 \mathrm{~km}^{2}$ kontrolliert und ausgewertet. Im Frühjahr zwischen 25. März und 30. Mai wurden an durch Zufallsmethodik im Forschungsareal ausgewählten Bäumen je zwei Äste an vier Seiten (Kompassrichtungen Süd, Ost, West und Nord) in 1,5-2 m Höhe (insgesamt 8 Äste) geschnitten und ins Labor gebracht. Außerdem wurden die Anzahl der Eier bzw. Eigelege pro Nadel und auch die Befallsstärke im Frühjahr festgestellt. Die Belegrate der Nadeln und die Eizahl je Eigelege wurden unter Verwendung einer Lupe bestimmt. Der Zusammenhang zwischen Nadellänge und Eierzahl im Gelege wurde ausgewertet.

Ebenso wurden in verschiedenen Bereichen des Untersuchungsareals befallene Äste im Freiland mit dünnen Stoffsäcken verschiedener Größe umhüllt. Auf diese Weise konnte der Parasitierungsgrad von Eiern und Larven festgestellt werden. Zur statistischen Untersuchung der Größenunterschiede der Kokons (Signifikanz einzelner Variablen und Durchschnittswerte) wurden die Faktorenanalyse und das T-Test Verfahren mit SPSS-Analyse verwendet.

Zur Schädlingsprognose wurde an 10 mit Kiefern bewachsenen Orten des Untersuchungsareals in $1 \mathrm{~m} \times 1 \mathrm{~m}\left(1 \mathrm{~m}^{2}\right)$ großen Flächen bis zu $5 \mathrm{~cm}$ tief im Boden gegraben und nach Kokons gesucht. Die gesammelten Kokons jeder Fläche wurden in separaten, mit Fliegendraht umspannten und mit etwas Erde befüllten Holzkäfigen im Labor gelagert. Zwischen dem 14. Juli und dem 3. August wurde Schlupfrate und Parasitierungsgrad der Käfige festgestellt. Aus den nicht geschlüpften Kokons wurden die Puppen im Labor herauspräpariert. Sie waren geschrumpft und abgestorben, höchstwahrscheinlich überliegende Puppen. Die Kokonmessungen wurden mit einer elektronischen digital-caliber Schieblehre $(0-150 \mathrm{~mm})$ realisiert. 


\section{Ergebnisse und Diskussion}

\subsection{Befallsbereich der Bäume und die Belegintensität der Nadel}

Die im Untersuchungsgebiet kontrollierten Bäume $(n=298)$ waren alle in unterschiedlichen Bereichen befallen, also waren keine Bäume vor dem Schädling verschont geblieben. Die Befallsstärke der Bäume ist in der Tabelle 1 zusammengestellt. Wie aus der Tab. 1 zu entnehmen ist, wurden 23,2 \% der Bäume kahlgefressen und 24,2 \% der Bäume zu 2/3 befallen.

Tab. 1: Befallsstärke der Kiefern durch D. pini (Anzahl der befallenen Kiefern = 298).

\begin{tabular}{|c|c|c|}
\hline Befallsklasse & Anzahl der Bäume & Anteil \\
\hline nur Kronenbereich befallen & 18 & $6,0 \%$ \\
\hline $1 / 4$ des Baumes befallen & 7 & $2,3 \%$ \\
\hline $1 / 3$ des Baumes befallen & 63 & $21,1 \%$ \\
\hline $1 / 2$ des Baumes befallen & 69 & $23,2 \%$ \\
\hline $2 / 3$ des Baumes befallen & 72 & $24,2 \%$ \\
\hline vollständig kahlgefressen & 69 & $23,2 \%$ \\
\hline
\end{tabular}

Die durchschnittliche Eizahl in einem Eigelege von $D$. pini an einer Nadel schwankt zwischen 1 und 43 (im Mittel 16,1 \pm 8,9) Eier.

Tab. 2: Ergebnisse der Untersuchnung der Eigelege von D. pini.

\begin{tabular}{|c|c|c|c|}
\hline \multicolumn{4}{|c|}{ Eizahl je Eigelege } \\
\hline Minimum & Maximum & Mittel & $\begin{array}{c}\text { Standard- } \\
\text { abweichung }\end{array}$ \\
\hline 1 & 43 & 16,1 & 8,9 \\
\hline
\end{tabular}

Tab. 3: Die Verteilung der Eierzahl auf den Nadeln mit unterschiedlichen Längen.

\begin{tabular}{|l|c|l|l|l|l|l|l|l|}
\hline & \multicolumn{7}{|c|}{ Anzahl der Eier pro Nadel } \\
\hline Nadellänge & $1-4$ & $5-7$ & $8-10$ & $11-15$ & $16-25$ & $26-30$ & $31-43$ & Summe \\
\hline $5,3-6,5 \mathrm{~cm}$ & 3 & 4 & 3 & 5 & 4 & & & 19 \\
& $15,8 \%$ & $21,1 \%$ & $15,8 \%$ & $26,3 \%$ & $21,1 \%$ & & & $100 \%$ \\
\hline $6,6-7,5 \mathrm{~cm}$ & 5 & 4 & 6 & 9 & 13 & 3 & & 40 \\
& $12,5 \%$ & $10,0 \%$ & $15,0 \%$ & $22,5 \%$ & $32,5 \%$ & $7,5 \%$ & & $100 \%$ \\
\hline $7,6-9,0 \mathrm{~cm}$ & 2 & 4 & 2 & 6 & 10 & 5 & 2 & 31 \\
& $6,5 \%$ & $12,9 \%$ & $6,5 \%$ & $19,4 \%$ & $32,3 \%$ & $16,1 \%$ & $6,5 \%$ & $100 \%$ \\
\hline $9,1-11,2 \mathrm{~cm}$ & & 1 & & 8 & 8 & 5 & 3 & 25 \\
& & $4,0 \%$ & & $32,0 \%$ & $32,0 \%$ & $20,0 \%$ & $12,0 \%$ & $100 \%$ \\
\hline Summe & 10 & 13 & 11 & 28 & 35 & 13 & 5 & 115 \\
& $8,7 \%$ & $11,3 \%$ & $9,6 \%$ & $24,3 \%$ & $30,4 \%$ & $11,3 \%$ & $4,3 \%$ & $100 \%$ \\
\hline
\end{tabular}


Wie aus Tabelle 3 zu entnehmen ist, enthalten 30,4\% aller Gelege 16-25 Eier, gefolgt von Gelegen mit 11-15 Eiern zu 24,3\%. Ob die Nadellänge diese Eizahlen beeinflusst hat, ist fraglich, weil die Weibchen Gelege mit 16-25 Eiern sowohl auf Nadeln der Längen 6,6-7,5 cm, 7,6-9,0 cm als auch 9,1-11,2 cm platziert hatten (Häufigkeiten 32,5 \%, 32,3 \% bzw. $32 \%$ ).

Zur Feststellung von Belegintensität und -rate wurden insgesamt an 2685 Nadel auf Eier untersucht. Davon waren nur 369 Nadel mit Eiern belegt. D. pini legte nur auf 13,7 \% der Nadel im Freiland Eier ab. Wie aus der Tabelle 4 zu entnehmen ist, hat $D$. pini während Sommergeneration in Beytepe 41,2 \% bzw. 18,0 \% der Nadel der Äst mit den Nummern 1 und 4 bevorzugt, die zu Bäumen am Südrand der Kulturfläche gehören.

Tab. 4: Belegrate der Nadel unter Freilandbedingungen.

\begin{tabular}{|c|c|c|c|}
\hline $\begin{array}{c}\text { Nummer } \\
\text { der Äste }\end{array}$ & $\begin{array}{c}\text { Anzahl } \\
\text { der Nadeln }\end{array}$ & $\begin{array}{c}\text { Anzahl der mit Eiern } \\
\text { belegten Nadeln }\end{array}$ & $\begin{array}{c}\text { Belegrate der Nadeln } \\
\text { in \% }\end{array}$ \\
\hline 1 & 277 & 114 & 41,2 \\
\hline 2 & 277 & 35 & 12,6 \\
\hline 3 & 430 & 38 & 8,8 \\
\hline 4 & 333 & 60 & 18,0 \\
\hline 5 & 378 & 49 & 13,0 \\
\hline 6 & 310 & 34 & 11,0 \\
\hline 7 & 437 & 36 & 8,2 \\
\hline 8 & 243 & 3 & 1,2 \\
\hline Summe & 2685 & 369 & 13,7 \\
\hline
\end{tabular}

\subsection{Schlupfrate und Parasitierungsgrad der männlichen und weiblichen Kokons und Geschlechteranteil}

Die Populationsgröße von $D$. pini in Beytepe kann mittels Zählung der Kokons prognostiziert werden. Ein massenhaftes Auftreten in der Folgegeneration ist zu erwarten, wenn 20 Kokons pro Quadratmeter gefunden werden (Mischprobe aus $10 \times 1 \mathrm{~m}^{2}$ Teilflächen). Um das Auftreten der Schädlinge zu prognostizieren, wurden auf 10 in verschiedenen Himmelsrichtungen des Untersuchungsgebietes verteilten Probeflächen von jeweils $1 \mathrm{~m}^{2}$ Größe insgesamt 270 Kokons der vorjährigen Generationen gesammelt und mit Erde ins Labor gebracht und beobachtet. Von zwei Stichproben konnte kein Kokon gewonnen werden, sie waren kokonfrei.

Mit durchschnittlich 27 Kokons von D. pini je $\mathrm{m}^{2}$ in Beytepe wäre der kritische Prognosewerte von 20 Kokons überschritten worden. Man hätte also mit einem Massenauftreten rechnen müssen. Aber in der Realität war dies nicht der Fall. Da 15,2 \% (N=41) der Kokons parasitiert waren und 12,2\% $(\mathrm{N}=33)$ nicht ausgeschlüpft sind, mussten sie bei der Berechnung des kritischen Prognosewertes nicht berücksichtigt werden. 
Tab. 5: Schlupf-, Parasitierungsrate und Weibchenanteil der Kokons von D. pini- Population in Beytepe - Ankara

\begin{tabular}{|c|c|c|c|c|c|c|c|c|c|c|c|c|c|c|}
\hline \multirow{3}{*}{$\begin{array}{l}\text { Nr. der } \\
\text { Stich- } \\
\text { probe } \\
\text { flä- } \\
\text { chen* }\end{array}$} & \multirow{3}{*}{$\begin{array}{l}\text { Anzahl } \\
\text { der } \\
\text { Kokons }\end{array}$} & \multicolumn{4}{|c|}{ ausgeschlüpft } & \multicolumn{4}{|c|}{ parasitiert } & \multicolumn{4}{|c|}{ nicht ausgeschlüpft } & \multirow{3}{*}{$\begin{array}{l}\text { Weib- } \\
\text { chen- } \\
\text { Anteil } \\
\text { in } \%\end{array}$} \\
\hline & & \multicolumn{2}{|c|}{ Weibchen } & \multicolumn{2}{|c|}{ Männchen } & \multicolumn{2}{|c|}{ Weibchen } & \multicolumn{2}{|c|}{ Männchen } & \multicolumn{2}{|c|}{ Weibchen } & \multicolumn{2}{|c|}{ Männchen } & \\
\hline & & $\mathrm{N}$ & $\%$ & $\mathrm{~N}$ & $\%$ & $\mathrm{~N}$ & $\%$ & $\mathrm{~N}$ & $\%$ & $\mathrm{~N}$ & $\%$ & $\mathrm{~N}$ & $\%$ & \\
\hline 1 & 60 & 31 & 51,7 & 8 & 13,3 & 9 & 15 & 1 & 1,7 & 8 & 13,3 & 3 & 5 & 80 \\
\hline 2 & 30 & 16 & 53,3 & 6 & 20 & 5 & 16,7 & - & - & 1 & 3,3 & 2 & 6,7 & 73,3 \\
\hline 3 & 30 & 17 & 56,7 & 5 & 16,7 & 4 & 13,3 & - & - & 4 & 13,3 & - & - & 83,3 \\
\hline 4 & 30 & 19 & 63,3 & 4 & 13,3 & 4 & 13,3 & - & - & 2 & 6,7 & 1 & 3,3 & 83,3 \\
\hline 5 & 30 & 15 & 50 & 7 & 23,3 & 4 & 13,3 & 1 & 3,3 & 1 & 3,3 & 2 & 6,7 & 66,7 \\
\hline 6 & 30 & 21 & 70 & 4 & 13,3 & 2 & 6,7 & - & - & 3 & 10 & - & - & 86,7 \\
\hline 7 & 30 & 20 & 66,7 & 3 & 10 & 4 & 13,3 & - & - & 2 & 6,7 & 1 & 3,3 & 86,7 \\
\hline 8 & 30 & 07 & 23,3 & 13 & 43,3 & 6 & 20 & 1 & 3,3 & 2 & 6,7 & 1 & 3,3 & 50,0 \\
\hline$S^{* *}$ & 270 & 146 & 54,1 & 50 & 18,5 & 38 & 14,1 & 3 & 1,1 & 23 & 8,5 & 10 & 3,7 & 76,7 \\
\hline
\end{tabular}

*=In zwei Stichproben waren keine Kokons zu finden, deswegen wurden nur 8 Proben in der Tabelle aufgeführt; $S^{* *}=$ Summe; $N$ = Anzahl der Kokons in den jeweiligen Stichproben.

In diesem Fall, wie aus der Tabelle 5 zu entnehmen ist, beträgt die Anzahl der ausgeschlüpften Kokons pro $\mathrm{m}^{2}$ Probefläche nur 19,6, was unter 20 Kokons liegt. Demnach ist ein Massenwechsel nicht zu erwarten (Bogenschütz u. a. 1982).

Dabei ist auch festzustellen, dass die Parasitierungswerte bei männlichen Kokons nur 1,1\% $(\mathrm{N}=3)$ und bei weiblichen Kokons 14,1\% $(\mathrm{N}=38)$ erreichten.

Es wurde auch der Geschlechteranteil in der Population festgestellt. Wie aus der Tabelle 5 zu entnehmen ist, sind von 270 Kokons 207 weiblich und 63 männlich. Also beträgt der Weibchenanteil $76,7 \%$. Wenn man aber die nichtausgeschlüpften bzw. parasitierten Kokons absieht, wird der Weibchenanteil 54,1\% ( $\mathrm{N}=146)$ betragen.

\subsection{Die Maße der weiblichen und männlichen Kokons und die Korrelation}

Dazu wurden Breite und Länge der weiblichen und männlichen Kokons der Beytepe-Population gemessen und in den Tabellen 6 und 7 zusammengestellt. Wie aus den Tabellen 6 und 7 zu entnehmen ist, unterscheiden sich männliche und weibliche Kokons mit einer Sicherheit von p>0,05 signifikant in ihrer Breite, nicht dagegen in ihrer Länge ( $p>0,05)$.

Die Gemeine Kiefernbuschhornblattwespe wird als ein wichtiger Forstschädling in türkischen Wäldern betrachtet (ACATAY 1969, ÇANAKÇıOĞLu 1998). Dieser Schädling kann im Kokon bis zu fünf Jahre überdauern (Schwenke 1964, Pschorn-Walcher 1982, EichHorn 1983a, 1983b, Auger-Rozenberg u. a. 1998). Das bedeutet, dass ausführliche biologische Untersuchung zu diesem Schädling in den türkischen Wäldern durchgeführt werden sollten. 
Tab. 6: Die Breite der weiblichen und männlichen Kokons von D. pini.

\begin{tabular}{|l|l|c|c|}
\hline \multicolumn{1}{|c|}{ Geschlechter } & \multicolumn{1}{c|}{ Anzahl der Kokons } & Mittel in cm & Standardabweichung \\
\hline Weibchen & 60 & 0,55 & 3,03 \\
\hline Männchen & 41 & 0,43 & 2,23 \\
\hline Geschlechter & & Weibchen & Männchen \\
\hline \multirow{4}{*}{ Weibchen } & Korrelation & 1,00 & $0,32^{*}$ \\
\cline { 2 - 5 } & Signifikant & - & 0,04 \\
\cline { 2 - 5 } & Anzahl & 60 & 41 \\
\hline \multirow{3}{*}{ Männchen } & Korrelation & $0,32^{*}$ & 1,00 \\
\cline { 2 - 5 } & Signifikant & 0,04 & - \\
\cline { 2 - 5 } & Anzahl & 41 & 41 \\
\hline
\end{tabular}

*) Unterschied ist mit einer Sicherheit von p>0,05 signifikant.

Tab. 7: Die Länge der weiblichen und männlichen Kokons von D. pini.

\begin{tabular}{|l|l|c|c|}
\hline \multicolumn{1}{|c|}{ Geschlechter } & \multicolumn{1}{c|}{ Anzahl der Kokons } & Mittel in cm & Standardabweichung \\
\hline Weibchen & \multicolumn{1}{c|}{60} & 1,05 & 0,10 \\
\hline Männchen & \multicolumn{1}{c|}{0,80} & 0,07 \\
\hline Geschlechter & & Männchen & Weibchen \\
\hline \multirow{3}{*}{ Weibchen } & Korrelation & $1,00^{*}$ & 0,04 \\
\cline { 2 - 4 } & Signifikant & - & 0,82 \\
\cline { 2 - 5 } & Anzahl & 60 & 41 \\
\hline \multirow{3}{*}{ Männchen } & Korrelation & 0,32 & 1,00 \\
\cline { 2 - 5 } & Signifikant & $0,82^{*}$ & - \\
\cline { 2 - 5 } & Anzahl & 41 & 41 \\
\hline
\end{tabular}

*) Der Unterschied zwischen männlichen und weiblichen Kokonlänge ist mit einer Sicherheit von p>0,05 nicht signifikant.

Das Untersuchungsareal in dieser Arbeit ist ein Kulturwald in Beytepe. Aus diesem Grund werden nur bestimmte biologische Gegebenheiten dieses Schädlings untersucht, mit der Annahme, dass hiesige Daten für weitere Untersuchungen etwas beitragen werden. Dieser Schädling kann in verschiedenen Massen die Bäume befallen, von den untersuchten Bäumen ( $\mathrm{n}=298)$ waren 14,0 \% kahl gefressen, was mit L̊̊ngSTRÖM u. a. (2001) übereinstimmt.

Die Weibchen hatten ihre Eier hauptsächlich an der Unterseite der Nadel abgelegt. Dabei hatten sie keine bestimmte Lage auf der Nadel vorgezogen. Gleiche Ergebnisse wurden von (PASQUIR u. a. 1999, 2000) gewonnen. Die mittlere Eizahl je Nadel und Gelege wurde in Beytepe mit 16,1 $\pm 8,9$ ermittelt, ähnliche Werte werden in Europa aus verschiedenen Gebieten gemeldet. Dagegen war die Belegrate der Nadel in verschiedenen Gebieten der Erde etwas größer als in Beytepe (Kangas 1963, Pschorn-Walcher 1982, Eichhorn 1983a, 1983b, Sharov u. a. 1993, Auger-Rozenberg u. a. 1998, LÅngström u. a. 2001). In Deutschland wurden bei einem starken Massenauftreten Kokondichten bis 100 Kokons je $\mathrm{m}^{2}$ festgestellt (PsCHORN-WALCHER 1982, EichHorn 1983a, 1983b, Herz, 1997, Herz u. a. 1999). Der kritische Wert zur Prognose im 
Massenwechsel dieses Schädlings liegt bei 20 Kokons je $\mathrm{m}^{2}$. Wird er überschritten, ist mit einem Massenauftreten des Schädlings in der Folgegeneration zu rechnen (Bogenschütz u. a. 1982, Pschorn-Walcher 1982, EichHorn 1983a, Herz u. a. 1999). In Beytepe erreichte die Population von $D$. pini diesen Wert nicht.

So ergaben die Bonituren in Beytepe eine Dichte von 27 Kokons je $\mathrm{m}^{2}$. Dennoch fand in den darauf folgenden zwei Jahren keine Massenvermehrung von D. pini in Beytepe statt. In der Populationsökologie spielt die Parasitierung verschiedener Stadien, wie Eier, Larven und Puppen, eine wichtige Rolle. Es ist sicher, dass die Larvenparasitierung bei der Reduzierung von D. piniPopulationen ein entscheidender Faktor sein kann (Herz \& Heitland 1999).

Die Parasitierung der Kokons ist methodisch leichter zu ermitteln. Mit unseren Untersuchungen konnten wir eine Parasitierungsrate von $15,2 \%$ der Kokons $(14,1 \%$ bei weiblichen $1,1 \%$ bei männlichen Kokons) feststellen. Das eine Massenvermehrung in den Folgejahren ausblieb, zeigt, wie groß der Einfluss der Puppenparasitierung auf den Massenwechsel ist. Das spricht für eine biologische Bekämpfung von $D$. pini, besonders, da viel höhere Parasitierungsraten bekannt sind. So berichtet Schwenke (1964) davon, dass die Hälfte bzw. Dreiviertel der Kokons von D. pini im Nürnberger Raum parasitiert waren.

Bekannt ist weiterhin, dass die Kokons im Untersuchungsgebiet während der Puppenruhe durch Waldmäuse, Apodemus sylvaticus, (Obrtel u. a. 1978, Hanski \& Parviainen 1985) und einige Vogelarten, z. B. der Kohlmeise, Parus major, vertilgt werden.

Die Maße der Kokons der weiblichen und männlichen Exemplare zeigen bestimmte Unterschiede. So sind die weiblichen Kokons statistisch gesichert breiter als die männlichen. Es steht ebenfalls fest, dass die kleineren männlichen Kokons weniger parasitiert (nur 1,1 \%) als die größeren weiblichen Kokons (14,1 \%) sind.

Zur Bekämpfung dieses Forst-Schädlings bei Massenvermehrung wurde der chemische Stoff Malathion verwendet. Da Malathion viele schwerwiegende Nebenwirkungen besonders an die Bienen und an Vögel anrichten kann, wurde es nur einmal angewendet, und dann nicht mehr wiederholt. Seit dem Jahre 2002 wurde im Untersuchungsgebiet kein wirtschaftlicher Schaden durch den Schädling registriert. In den darauf folgenden Jahren bis heute trat er in so geringem Maße auf, dass man nicht bekämpfen musste.

Zur Kontrolle der Populationsdynamik von D. pini, insbesondere in solchen Latenzphasen, bieten sich biologische Maßnahmen an. Aus diesem Grund müssen insektivore Vögel durch Anbringung der Kunstnistkästen in den Wäldern der Türkei unterstützt und geschützt werden (KızıroĞLu 2005, KiziroĞLu u. a. 2002, Turan u. a. 2003, ErdoĞAn u. a. 2005).

Auch andere biologische Bekämpfungsstrategien gegen diesen und verwandte Schädlinge sind möglich. Viren gegen Neodiprion sertifer (Geofrroy, 1785) und Pheromone gegen manche wichtige Forstschädlinge zeigen gleichzeitig Wirkung auf D. pini (Podgwaite u. a. 1984, Anderbrant u. a. 1995, Bergström u. a. 1995, LÅngstrom u. a. 2001, YAmAn u. a. 2001, JohAnsson u. a. 2002, ŞıMŞEK 2004).

\section{Literatur}

AcataY, G. 1969: Tatbiki Orman Entomolojisi. - İstanbul Üniversitesi Orman Fakültesi Yayin. No: 1359; Orman Fakültesi No: 133, Istanbul, 182 pp.

Anderbrant, O.; Hansson, B. S.; Hallberg, E.; Geri, C.; Varama, M.; Hedenström, E.; Högberg, H. E.; Fägerhag, J.; Edlund, E.; Wassgren, A. B.; Bergström, G. \& ÖfQvist, J. L. 1995: Electrophysilogical and morphological characteristics of pheromone receptors in male pine sawflies, Diprion pini, and behavioural response to some compounds. - Journal of Insect Physiology 41: 395-401. 
Auger-Rozenberg, M.-A.; Geri, C.; Quencez C. \& Bastein, C. 1998: Noxius Effect of scots foliage on Diprion pini L. (Hymenoptera, Diprionidae) and other defoliators: Specifity and Prospects for use in Forest Management. - In: M. L. McManus and A. M. Liebhold (Ed.) USDA Forest Service General Technical Report NE - 247: 91-99.

Bergström, G.; Wassgren, A. B.; Anderbrant, O.; Fägerhag, J.; Edlund, H.; Hedenström, E.; Högberg, H. E.; Geri, C.; Varama, M.; Hansson, B. S. \& LöfQvist, J. 1995: Sex pheromone of the pine sawfly Diprion pini, chemical identification, synthesis and biological activity. - Experientia 51: 370-380.

Bogenschütz, H. \& KönIG, E. 1982: Die Gemeine Kiefernbuschhornblatwespe (Diprion pini L.). - Merkblätter der forstlichen Versuchs- und Forschungsanstalt Baden-Württemberg, Nr. 27: 7 pp.

ÇANAKÇıOĞLU,H. 1998: Orman Entomolojisi. - Zararlı ve Yararlı Böcekler. İstanbul Üniversitesi Orman Fakültesi Yayini: 541 pp.

Eichнorn, O. 1980: Autökologische Untersuchungen an Populationen der gemeinen Kiefern-Buschhornblattwespe Diprion pini (L.) (Hymenoptera: Diprionidae). V. Untersuchungen über die larvenparasitischen Tachinen, insbesondere über Drino gilva HTG. - Zeitschrift für angewandte Entomologie 89: 455-470.

Eichнorn, O. 1981: Autökologische Untersuchungen an Populationen der Gemeinen Kiefern-Buschhornblattwespe Diprion pini (L.) (Hymenoptera: Diprionidae). VI. Zur Kenntnis der Kokonparasiten und Exenterus-Arten. - Zeitschrift für angewandte Entomologie 92: 252-285.

Eichnorn, O. 1983a: Dormanzverhalten der Gemeinen Kiefern-Buschhornblattwespe (Diprion pini L.) (Hymenoptera, Diprionidae) und ihrer Parasiten. - Zeitschrift für angewandte Entomologie 95: 482-498.

EichHoRn, O. 1983b: Untersuchungen zur Ökologie der Gemeinen Kiefern-Buschhornblattwespe, Diprion pini (L.) (Hymenoptera, Diprionidae). VIII. Verlauf der Massenvermehrung bei Speyer (Pfalz) 1976-1978. - Zeitschrift für angewandte Entomologie 96: 291-303.

ErdoĞan, A.; KaÇar, M. S.; Turan, L.; KiziroĞLu, I. \& Öz, M. 2005: Forest pest control by Common Redstart (Phoenicurus phoenicurus L.) near Antalya, Turkey. - Journal of Pesticide Science 78 (July 2005): 46.

Hanski, I. \& Parviainen P. 1985: Cocoon predation by small mammals and pine sawfly population dynamics. - OIKOS 54: 125-136.

Herz, A. 1997: Zur Effektivität und Struktur der Parasitoidengemeinschaft von Diprion pini (L.) (Hymenoptera: Diprionidae) bei latenten Dichten des Wirtes. - PhD thesis, Ludwigs-Maximillians-Universität München: 193 pp.

Herz, A. \& Heitland, W. 1999: Larval parasitism of a forest pest, the common pine sawfly Diprion pini L. (Diprionidae), during an endemic density phase. - Journal of Applied Entomology 123: 129-137.

Johansson, B. G.; Anderbrant, O. \& Sierpinski, A. 2002: Multispecies trapping of six pests of scots pine in Sweden and Poland. - Journal of Applied Entomology 126: 212-216.

Kangas, E. 1963: Über das schädliche Auftreten der Diprion-Arten in finnischen Kiefernbeständen in diesem Jahrhundert. - Zeitschrift für angewandte Entomologie 51: 188-194.

KızıroĞLU, İ. 2005: Genel Biyoloji. 5. Basim (Auflage). - Birlik Matbaasi Ankara: 722 pp.

KiziroĞLu, I.; Turan, L.; ErdoĞan, A.; Adizel, Ö. \& PamukoĞLu, N. 2002: Zur Schädlingsvertilgung einiger Singvogelarten in einem Schwarzkiefern/Eichen-Mischbestand im Beynamer Wald bei Ankara. - Anzeiger für Schädlingskunde, Pflanzenschutz, Umweltschutz (Journal of Pest Science) 75: 99-102.

Långström, B.; Annila, E.; Hellqvist, C.; Varama, M. \& Niemela, P. 2001: Tree Mortality, Needle Biomass Recovery and Growth Losses in Scots Pine Following Defoliation by Diprion pini (L.) and Subsequent Attack by Tomicus piniperda (L.). - Scandinavian Journal of Forest Research 16: 342-353.

Obrtel, R.; ZejDa, J. \& Holisova, V. 1978: Impact of small rodent predation on an overcrowded population of Diprion pini during winter. - Folia Zoologica 27: 97-110.

Pasquier, B. F.; Rozenberg, M. A.; Lieutier F. (Ed.); Mattson, WJ. (Ed.) \& Wagner, Mr. 1999: Effects of foliage quality of different Pinus sylvestris L. Clones on Diprion pini L. Biology (Hymenoptera, Diprionidae). - Physiology and genetics of tree-phytophage interactions. International Symposium, Gujan, France, 31 August - 5 September, 1997: 211-219; Les Colloques No. 90; INRA Editions; Versailles; France; INRA, Station de Zoologie Forestiere, 45160 Olivet, France. 
Pasquier, B. F.; Geri, C.; Goussard, F.; Rozenberg, M. A. \& Grenier, S. 2000: Oviposition preference and larval survival of Diprion pini on Scots pine clones in relation to foliage characteristics. - Agricultural and Forest Entomology 2 (3): 185-192.

Podgwaite, J. D.; Rush, P.; Hall, D. \& Walton G. S. 1984: Efficacy of the Neodiprion sertifer (Hymenoptera: Diprionidae) nucleopolyhydrosis virus (baculovirus) product, neochek. S. J. - Ecologocal Entomology 77: 525-528.

Pschorn-Walcher, H. 1982: Unterordnung Symphyta. Pflanzenwespen. - In: Schwenke, W. (ed.): Die Forstschädlinge Europas. 4. Bd. Hautflügler und Zweiflügler. Pp. 4-196. Paul Parey, Berlin.

Sharov, A. A.; Wagner, M. R. (Ed.) \& Raffa, K. F. 1993: Biology and population dynamics of the common pine sawfly, Diprion pini L., in Russia. Academic Press, Inc.; San Diego; USA. Forest Service, Morgentown, WV, USA.

Schwenke, W. 1960: Über die Wirkung der Walddüngung auf die Massenvermehrung der Kiefernbuschhornblattwespe (Diprion pini L.)1959 in Mittelfranken und die hieraus ableitbaren gradologischen Folgerungen. - Zeitschrift für angewandte Entomologie 46: 371-378.

Schwenke, W. 1964: Grundzüge der Populationsdynamik und Bekämpfung der gemeinen KiefernbuschhornBlattwespe, Diprion pini L. - Zeitschrift für angewandte Entomologie 54: 101-107.

ŞıмşeK, Z. 2004: Kitin Sentezini Engelleyen Diflubenzuron İlacının Sarıçamın Önemli Zararlısı Olan Çalı Antenli Çam Yaprakarısı (Diprion pini L., Hymenoptera; Diprionidae) Mücadelesinde Kullanım İmkanlarının Araştırılması. - Süleyman Demirel Üniversitesi, Orman Fakültesi Dergisi Seri: A, Sayı: 2: 48-59.

Thalenhorst, W. 1952: Das Auftreten von Kiefernbuschhornblattwespe in Norddeutschland 1949. Zeitschrift für angewandte Entomologie 34: 45-64.

Thalenhorst, W. 1954: Vergleichende Betrachtungen über den Massenwechsel der Kiefernbuschhornblattwespen. - Zeitschrift für angewandte Entomologie 35: 168-182.

Turan, L. \& Kiziroglu, İ. 2003: Die Schädlingsvertilgung einiger Singvogelarten in einem SchwarzkieferZedern-Wald in Beytepe bei Ankara, Türkei. Preying of some species of singing birds in ablack pinecedar-forest in Beytepe near Ankara. - Anzeiger für Schädlingskunde, Pflanzenschutz, Umweltschutz (Journal of Pest Science) 76 (6): 159-160.

Urban, S. 1965: Die Begrenzungsfaktoren der Übervermehrung der Kiefernbuschhornblattwespe (Diprion pini L.) in den Jahren 1960 und 1961. (Eine Ergänzung der Prognose des Blattwespen-Auftretens.) - Archiv Forstwesen 14: 1223-1234.

Yaman, M.; NalçacioĞLu, R. \& DemirbaĞ, Z. 2001: Viral control of the European Pine Sawfly, Neodiprion sertifer (Geoffroy) in Turkey. - Turkish Journal of Biology 25: 419-425.

\section{Anschrift der Verfasser:}

Prof. Dr. Ilhami KiziroĞLu und Prof. Dr. Levent Turan

Hacettepe Universitaet, Naturwissenschaftliche Abteilung

Didaktik der Biologie

06530 Beytepe / Ankara

Türkei

e-mail: ikiziroglu@gmail.com

\section{Subject editor:}

Dr. A. TAEger 